\title{
Observational constraints on Myrzakulov gravity
}

\author{
Fotios K. Anagnostopoulos ${ }^{a}$ Spyros Basilakos ${ }^{b, c}$ Emmanuel N. Saridakis $^{c, d, e}$ \\ ${ }^{a}$ Department of Physics, National \& Kapodistrian University of Athens, Zografou Campus GR 157 73, \\ Athens, Greece \\ ${ }^{b}$ Academy of Athens, Research Center for Astronomy and Applied Mathematics, Soranou Efesiou 4, 11527 , \\ Athens, Greece \\ ${ }^{c}$ National Observatory of Athens, Lofos Nymfon, 11852 Athens, Greece \\ ${ }^{d}$ CAS Key Laboratory for Researches in Galaxies and Cosmology, Department of Astronomy, University of \\ Science and Technology of China, Hefei, Anhui 230026, P.R. China \\ ${ }^{e}$ School of Astronomy, School of Physical Sciences, University of Science and Technology of China, Hefei \\ 230026, P.R. China \\ E-mail: fotis-anagnostopoulos@hotmail.com, \\ svasileacademyofathens.gr, msaridakenoa.gr
}

\begin{abstract}
We use data from Supernovae (SNIa) Pantheon sample, from Baryonic Acoustic Oscillations (BAO), and from cosmic chronometers measurements of the Hubble parameter (CC), alongside arguments from Big Bang Nucleosynthesis (BBN), in order to extract constraints on Myrzakulov $F(R, T)$ gravity. This is a connection-based theory belonging to the Riemann-Cartan subclass, that uses a specific but non-special connection, which then leads to extra degrees of freedom. Our analysis shows that both considered models lead to $\sim 1 \sigma$ compatibility in all cases. For the involved dimensionless parameter we find that it is constrained to an interval around zero, however the corresponding contours are slightly shifted towards positive values. Furthermore, we use the obtained parameter chains so to reconstruct the corresponding Hubble function, as well as the dark-energy equation-of-state parameter, as a function of redshift. As we show, Model 1 is very close to $\Lambda \mathrm{CDM}$ scenario, while Model 2 resembles it at low redshifts, however at earlier times deviations are allowed. Finally, applying the AIC, BIC and the combined DIC criteria, we deduce that both models present a very efficient fitting behavior, and are statistically equivalent with $\Lambda$ CDM cosmology, despite the fact that Model 2 does not contain the latter as a limit.
\end{abstract}

KEYWORDS: Modified gravity, Observational Constraints, Torsional Gravity 


\section{Contents}

1 Introduction 1

2 Myrzakulov Gravity and Cosmology 2

2.1 Myrzakulov Gravity 2

2.2 Cosmology 4

2.2.1 Model $1 \quad 5$

2.2.2 Model 2 6

3 Data and Methodology $\quad 7$

$\begin{array}{lll}3.1 \text { Cosmological probes } & 7\end{array}$

3.1.1 Direct measurements of the Hubble expansion 7

$\begin{array}{lll}3.1 .2 & \text { Supernovae Type Ia } & 7\end{array}$

3.1.3 Baryonic Acoustic Oscillations $\quad 8$

3.1.4 Big Bang Nucleosynthesis 9

3.1.5 Joint likelihood analysis 9

$\begin{array}{ll}3.2 & \text { Information Criteria and Model Selection } \\ \end{array}$

4 Results 11

5 Conclusions 16

\section{Introduction}

According to the concordance cosmological model the universe experienced two epochs of accelerated expansion, one at early and one at late times. Although the latter can be explained by the presence of a cosmological constant, the related theoretical problem, the possibility of a dynamical behavior, and especially the inability of the cosmological constant to describe the early accelerated phase, led to the incorporation of some form of modification. As a first possibility one can maintain general relativity as the underlying theory and modify the matter content of the universe by introducing extra fields, such as the inflaton at early times [1,2] and/or the dark energy sector at late times $[3,4]$. As a second possibility one modifies the gravitational sector itself, constructing a theory that possesses general relativity as a particular limit but which in general exhibits extra degrees of freedom [5, 6].

There are many ways to construct gravitational modifications, each one modifying a particular feature of general relativity. Modifying the dimensionality gives rise to the braneworld theories [7], modifying the Einstein-Hilbert Lagrangian, gives rise to $F(R)$ gravity [8, 9], $F(G)$ gravity $[10,11]$, Lovelock theories $[12,13]$, etc, while adding a scalar field coupled with curvature in various ways gives rise to Horndeski/Galileon theories [14-16]. Additionally, starting from the 
equivalent, teleparallel, formulation of gravity $[17,18]$ one can construct modifications using torsional invariants, such as in $F(T)$ gravity [19, 20], in $F\left(T, T_{G}\right)$ gravity [21], or in scalar-torsion theories [22, 23]. Moreover, one can construct the general class of metric-affine theories [24-26], which incorporates a general linear connection structure, or proceed to the introduction of nonlinear connections such as in in Finsler and Finsler-like theories [27-32].

Inspired by these, one could start from such affinely connected metric theories, and in particular from their Riemann-Cartan subclass [33], and construct a theory using a specific but non-special connection, which would lead to non-zero torsion and non-zero curvature at the same time, and thus offering the extra degrees of freedom typically needed in any gravitational modification [34]. Myrzakulov gravity can thus lead to a good phenomenology, being able to describe the universe evolution at early and late times [35-39].

One basic question in modified gravities is the determination of the arbitrary function that enters in the theory. Although some general features can be deduced through theoretical considerations, such as the absence of ghosts and instabilities, or the existence of Noether symmetries, the most powerful tool is the use of observational data [40-60]. Hence, in this work we are interested in using expansion data such as Supernovae type Ia data (SNIa), Baryonic Acoustic Oscillations (BAO), and Hubble Cosmic Chronometers (CC) observations, in order to impose constraints on Myrzakulov gravity. The plan of the work is the following. In Section 2 we present Myrzakulov gravity and its cosmological applications. In Section 3 we describe the various datasets and the involved statistical methods. Then in Section 4 we preform our analysis and we present the results, namely the constraints on the various parameters. Finally, in Section 5 we summarize and conclude.

\section{Myrzakulov Gravity and Cosmology}

In this section we present a brief review of Myrzakulov gravity, or $F(R, T)$ gravity [34, 35], extracting additionally the relevant cosmological equations.

\subsection{Myrzakulov Gravity}

The central idea of this modified gravity is the modification of the underlying connection. In particular, it is known that imposing a general connection $\omega_{b c}^{a}$ one defines the curvature and the torsion tensor respectively as [21]

$$
\begin{gathered}
R_{b \mu \nu}^{a}=\omega_{b \nu, \mu}^{a}-\omega_{b \mu, \nu}^{a}+\omega_{c \mu}^{a} \omega_{b \nu}^{c}-\omega^{a}{ }_{c \nu} \omega_{b \mu}^{c}, \\
T^{a}{ }_{\mu \nu}=e^{a}{ }_{\nu, \mu}-e^{a}{ }_{\mu, \nu}+\omega^{a}{ }_{b \mu} e^{b}{ }_{\nu}-\omega_{b \nu}^{a} e_{\mu}^{b},
\end{gathered}
$$

where $e_{a}^{\mu} \partial_{\mu}$ is the tetrad field related to the metric through $g_{\mu v}=\eta_{a b} e^{a}{ }_{\mu} e^{b}{ }_{v}$, where $\eta_{a b}=$ $\operatorname{diag}(1,-1,-1,-1)$, with Greek and Latin indices denoting coordinate and tangent space respectively, and where comma denotes differentiation.

There are infinite connection choices. The Levi-Civita $\Gamma_{a b c}$ is the only connection that gives vanishing torsion, and from now on we use the label "LC" to denote the curvature (Riemann) tensor corresponding to $\Gamma_{a b c}$, namely $R_{b \mu \nu}^{(L C) a}=\Gamma_{b v, \mu}^{a}-\Gamma_{b \mu, v}^{a}+\Gamma_{c \mu}^{a} \Gamma_{b v}^{c}-\Gamma_{c \nu}^{a} \Gamma_{b \mu}^{c}$. On the other hand one can use the Weitzenböck connection $W_{\mu \nu}^{\lambda}=e_{a}^{\lambda} e^{a}{ }_{\mu, v}$ which is curvatureless, leading only to 
torsion as $T_{\mu \nu}^{(W) \lambda}=W_{\nu \mu}^{\lambda}-W_{\mu \nu}^{\lambda}$ (we use the label "W" to denote quantities corresponding to $W_{\mu \nu}^{\lambda}$. From the above it is implied that the Ricci scalar corresponding to the Levi-Civita connection is

$$
R^{(L C)}=\eta^{a b} e_{a}^{\mu} e_{b}{ }^{v}\left[\Gamma_{\mu \nu, \lambda}^{\lambda}-\Gamma_{\mu \lambda, v}^{\lambda}+\Gamma_{\mu \nu}^{\rho} \Gamma_{\lambda \rho}^{\lambda}-\Gamma_{\mu \lambda}^{\rho} \Gamma_{\nu \rho}^{\lambda}\right],
$$

while the torsion scalar corresponding to the Weitzenböck connection is

$$
\begin{aligned}
T^{(W)}= & \frac{1}{4}\left(W^{\mu \lambda \nu}-W^{\mu \nu \lambda}\right)\left(W_{\mu \lambda \nu}-W_{\mu \nu \lambda}\right)+\frac{1}{2}\left(W^{\mu \lambda \nu}-W^{\mu \nu \lambda}\right)\left(W_{\lambda \mu \nu}-W_{\lambda \nu \mu}\right) \\
& -\left(W_{v}{ }^{\mu \nu}-W_{v}{ }^{\nu \mu}\right)\left(W_{\mu \lambda}^{\lambda}-W_{\lambda \mu}^{\lambda}\right) .
\end{aligned}
$$

As it is known, the former is used in the Lagrangian of General Relativity and in all curvature-based modified gravities, e.g. in $F(R)$ gravity [8], while the latter is used in the Lagrangian of teleparallel equivalent of general relativity and in all torsion-modified gravities, e.g. in $F(T)$ gravity [6].

In Myrzakulov gravity one uses a non-special connection which has non-zero curvature and torsion simultaneously [35]. Hence, the resulting theory will in general possess extra degrees of freedom, even if the imposed Lagrangian is simple, which is not the case of general relativity or of teleparallel equivalent of general relativity that both have two degrees of freedom corresponding to the massless graviton. The action of the theory is

$$
S=\int d^{4} x e\left[\frac{F(R, T)}{2 \kappa^{2}}+L_{m}\right],
$$

where $e=\operatorname{det}\left(e_{\mu}^{a}\right)=\sqrt{-g}, \kappa^{2}=8 \pi G$ is the gravitational constant, and where we have introduced the matter Lagrangian $L_{m}$ too for completeness. Note that in the arbitrary function $F(R, T)$ the $R$ and $T$ are the curvature and torsion scalars corresponding to the non-special connection used, which read as [21]

$$
\begin{aligned}
T & =\frac{1}{4} T^{\mu \nu \lambda} T_{\mu \nu \lambda}+\frac{1}{2} T^{\mu \nu \lambda} T_{\lambda \nu \mu}-T_{\nu}{ }^{\nu \mu} T_{\lambda \mu}^{\lambda}, \\
R & =R^{(L C)}+T-2 T_{\nu}{ }^{\nu \mu} ; \mu
\end{aligned}
$$

where ; marks the covariant differentiation with respect to the Levi-Civita connection. Therefore, $T$ depends on the tetrad field, its first derivative and the connection, while $R$ depends on the tetrad and its first derivative, and on the connection and its first derivative, with an additional dependence on the second tetrad derivative due to the last term of (2.7). Thus, using (2.4),(2.6),(2.7) we can finally write

$$
\begin{aligned}
& T=T^{(W)}+v, \\
& R=R^{(L C)}+u,
\end{aligned}
$$

where $v$ is a scalar depending on the tetrad, its first derivative and the connection, while $u$ is a scalar depending on the tetrad, its first and second derivatives, and the connection and its first derivative.

The quantities $u$ and $v$ quantify the effect of the specific but non-special imposed connection. In the case where this connection becomes the Levi-Civita one, then $u=0$ and $v=-T^{(W)}$, and the above theory becomes the usual $F(R)$ gravity, which in turn coincides with general relativity under $F(R)=R$. On the other hand, in the case where the connection is the Weitzenböck one, then we have that $v=0$ and $u=-R^{(L C)}$ and hence the theory coincides with $F(T)$ gravity, which in turn becomes the teleparallel equivalent of general relativity for $F(T)=T$. 


\subsection{Cosmology}

Let us now apply the above into a cosmological framework and extract the corresponding equations that determine the universe evolution. As it was shown in [35], in order to avoid complications related to the additional variation in terms of the connection, it proves convenient to apply a minisuper-space procedure. Hence, we apply the homogeneous and isotropic flat Friedmann-RobertsonWalker (FRW) geometry

$$
d s^{2}=d t^{2}-a^{2}(t) \delta_{i j} d x^{i} d x^{j}
$$

which corresponds to the tetrad $e^{a}{ }_{\mu}=\operatorname{diag}[1, a(t), a(t), a(t)]$, where $a(t)$ is the scale factor. In this case one can easily find that $R^{(L C)}=6\left(\frac{\ddot{a}}{a}+\frac{\dot{a}^{2}}{a^{2}}\right)$ and $T^{(W)}=-6\left(\frac{\dot{a}^{2}}{a^{2}}\right)$. Furthermore, we use the standard replacement $L_{m}=-\rho_{m}(a)$ [61-63]. Lastly, following the discussion on the dependence of $u$ and $v$ above, we consistently impose that $u=u(a, \dot{a}, \ddot{a})$ and $v=v(a, \dot{a})$.

In this work we are interested in exploring the cosmological behavior that arise purely from the non-special connection of Myrzakulov gravity. Hence, we focus on the simplest case where the involved arbitrary function is trivial, namely $F(R, T)=R+\lambda T$ with $\lambda$ a dimensionless parameter (we omit the coupling coefficient of $R$ since it can be absorbed into $\kappa^{2}$ ). Note that we do not consider an explicit cosmological constant term in the Lagrangian. Inserting the above mini-superspace expressions into the action (2.5), for this Lagrangian choice we acquire $S=\int L d t$, where

$$
L=\frac{3}{\kappa^{2}}[\lambda+1] a \dot{a}^{2}-\frac{a^{3}}{2 \kappa^{2}}[u(a, \dot{a}, \ddot{a})+\lambda v(a, \dot{a})]+a^{3} \rho_{m}(a) .
$$

Extracting the equations of motion for $a$, alongside the Hamiltonian constraint $\mathcal{H}=\dot{a}\left[\frac{\partial L}{\partial \dot{a}}-\frac{\partial}{\partial t} \frac{\partial L}{\partial \ddot{a}}\right]+$ $\ddot{a}\left(\frac{\partial L}{\partial \ddot{a}}\right)-L=0$, we finally acquire the Friedmann equations

$$
\begin{aligned}
3 H^{2} & =\kappa^{2}\left(\rho_{m}+\rho_{d e}\right) \\
2 \dot{H}+3 H^{2} & =-\kappa^{2}\left(p_{m}+p_{d e}\right),
\end{aligned}
$$

where

$$
\begin{aligned}
& \rho_{d e}=\frac{1}{\kappa^{2}} {\left[\frac{H a}{2}\left(u_{\dot{a}}+v_{\dot{a}} \lambda\right)-\frac{1}{2}(u+\lambda v)+\frac{a u_{\ddot{a}}}{2}\left(\dot{H}-2 H^{2}\right)-3 \lambda H^{2}\right] } \\
& p_{d e}=-\frac{1}{\kappa^{2}} {\left[\frac{H a}{2}\left(u_{\dot{a}}+v_{\dot{a}} \lambda\right)-\frac{1}{2}(u+\lambda v)-\frac{a}{6}\left(u_{a}+\lambda v_{a}-\dot{u}_{\dot{a}}-\lambda \dot{v}_{\dot{a}}\right)\right.} \\
&\left.-\frac{a}{2}\left(\dot{H}+3 H^{2}\right) u_{\ddot{a}}-H a \dot{u}_{\ddot{a}}-\frac{a}{6} \ddot{u}_{\ddot{a}}-\lambda\left(2 \dot{H}+3 H^{2}\right)\right],
\end{aligned}
$$

with $H=\frac{\dot{a}}{a}$ the Hubble parameter, $p_{m}$ the matter pressure, and with the subscripts $a, \dot{a}, \ddot{a}$ denoting partial derivatives with respect to this argument. Hence, in the theory at hand, we obtain an effective dark energy sector which arises from the non-special connection. Additionally, given the matter conservation equation $\dot{\rho}_{m}+3 H\left(\rho_{m}+p_{m}\right)=0$ we find

$$
\dot{\rho}_{d e}+3 H\left(\rho_{d e}+p_{d e}\right)=0,
$$

which implies that the effective dark energy sector is conserved. 
The above Friedmann equations can efficiently describe the late-time acceleration. A first observation is that in the case where $\lambda=0$, namely in the case where the Lagrangian is just the curvature (nevertheless the non-special connection leads to non-zero torsion too), and for the choice $u=c_{1} \dot{a}-c_{2}$, with $c_{1}, c_{2}$ constants, then we have $\rho_{d e}=-p_{d e}=\frac{c_{2}}{2 \kappa^{2}} \equiv \Lambda$. Hence, the scenario at hand includes $\Lambda \mathrm{CDM}$ cosmology as a sub-case, although we have not considered an explicit cosmological constant, since the cosmological constant arises effectively from the connection structure of the theory. Thus, we expect that a realistic model would be a deviation from the above scenario.

Finally, it proves convenient to introduce the decceleration parameter as

$$
q=-1-\frac{\dot{H}}{H^{2}}
$$

which quantifies the cosmic acceleration. Defining additionally the density parameters $\Omega_{m}=$ $\kappa^{2} \rho_{m} /\left(3 H^{2}\right)$ and $\Omega_{d e}=\kappa^{2} \rho_{d e} /\left(3 H^{2}\right)$, as well as the equation-of-state parameters $w_{m} \equiv p_{m} / \rho_{m}$ and $w_{d e} \equiv p_{d e} / \rho_{d e}$, we can extract the useful expression

$$
\frac{2 q-1}{3}=\Omega_{m} w_{m}+\Omega_{d e} w_{d e} .
$$

Hence, in the standard case of dust matter, namely for $w_{m} \approx 0$, we obtain

$$
w_{d e}=\frac{2 q-1}{3\left(1-\Omega_{m}\right)} .
$$

This expression allows one to find the evolution of the dark energy equation-of-state parameter, knowing the solution of the Friedmann equations, or knowing the observable values of $H(z)$ (where $z$ is the redshift defined through $1+z=a_{0} / a$ setting the current value of the scale factor to $a_{0}=1$ ).

In the following we will focus to two models which can satisfy these features.

\subsubsection{Model 1}

Choosing $u=c_{1} \dot{a}-c_{2}$ and $v=c_{3} \dot{a}-c_{4}$, with $c_{3}, c_{4}$ constants, we obtain

$$
\begin{aligned}
3 H^{2} & =\kappa^{2}\left(\rho_{m}+\rho_{d e}\right) \\
2 \dot{H}+3 H^{2} & =-\kappa^{2}\left(p_{m}+p_{d e}\right),
\end{aligned}
$$

with

$$
\begin{aligned}
\rho_{d e} & =\frac{1}{\kappa^{2}}\left[c-3 \lambda H^{2}\right] \\
p_{d e} & =-\frac{1}{\kappa^{2}}\left[c-\lambda\left(2 \dot{H}+3 H^{2}\right)\right],
\end{aligned}
$$

where $c \equiv c_{2}+c_{4}$. Hence, in this scenario the geometrical sector constitutes an effective dark energy sector with the above energy density and pressure, and an equation-of-state parameter of the form

$$
w_{d e}=-1+\frac{2 \lambda \dot{H}}{c-3 \lambda H^{2}} .
$$

Interestingly enough, we can see that $w_{d e}$ can be both larger or smaller than -1 , and thus the effective dark energy can be quintessence or phantom like. 
This model has two parameters, namely $c, \lambda$, but one of them can be eliminated using the present value of the matter density parameter $\Omega_{m 0}$ (from now on the subscript "0" denotes the current value of a quantity), since (2.20) at present gives:

$$
1=\Omega_{m 0}+\frac{c}{3 H_{0}^{2}}-\lambda
$$

Additionally, the deceleration parameter (2.18), using (2.22),(2.23), becomes

$$
q(z)=-1+\frac{2 \Omega_{m 0}(1+z)^{3}}{\Omega_{m 0}(1+z)^{3}+2\left(1+\lambda-\Omega_{m 0}\right)},
$$

and thus its value at present is

$$
q_{0}=-1+\frac{3 \Omega_{m 0}}{2(1+\lambda)}
$$

Comparing with the corresponding value of $\Lambda$ CDM scenario, namely $q_{0}^{\Lambda}=-1+3 \Omega_{m 0} / 2$, we verify that for the special case of $\lambda=0$ the two scenarios coincide, as mentioned above. Finally, note that from relation (2.26) we can calculate the transition redshit, namely the redshift in which $q$ transits from posotive to negative and we have the onset of acceleration, finding

$$
z_{t r}=-1+2^{1 / 3}\left(1+\lambda-\Omega_{m 0}\right)^{1 / 3} \Omega_{m 0}^{-1 / 3} .
$$

\subsubsection{Model 2}

As a second example let us consider a more general model with $u=c_{1} \frac{\dot{a}}{a} \ln \dot{a}$ and $v=s(a) \dot{a}$, with $s(a)$ an arbitrary function. In this case (2.14),(2.15) give

$$
\begin{aligned}
3 H^{2} & =\kappa^{2}\left(\rho_{m}+\rho_{d e}\right) \\
2 \dot{H}+3 H^{2} & =-\kappa^{2}\left(p_{m}+p_{d e}\right),
\end{aligned}
$$

with

$$
\begin{aligned}
& \rho_{d e}=\frac{1}{\kappa^{2}}\left[\frac{c_{1}}{2} H-3 \lambda H^{2}\right] \\
& p_{d e}=-\frac{1}{\kappa^{2}}\left[\frac{c_{1}}{2} H+\frac{c_{1}}{6} \frac{\dot{H}}{H}-\lambda\left(2 \dot{H}+3 H^{2}\right)\right],
\end{aligned}
$$

while

$$
w_{d e}=-1+\frac{2 \lambda \dot{H}-\frac{c_{1}}{6} \frac{\dot{H}}{H}}{\frac{c_{1}}{2} H-3 \lambda H^{2}} .
$$

Similarly to the previous example, for this case too $w_{d e}$ can be quintessence-like or phantom-like.

This model has two parameters, namely $c_{1}, \lambda$, but one of them can be eliminated using $\Omega_{m 0}$, since (2.20) at present time leads to:

$$
1=\Omega_{m 0}+\frac{c_{1}}{6 H_{0}}-\lambda
$$

The deceleration parameter (2.18) becomes

$$
q(z)=-1+\frac{\frac{3}{2} \Omega_{m 0}(1+z)^{3}}{3\left(1-\Omega_{m 0}+\lambda\right)+(1+\lambda)^{-1}\left[\left(1-\Omega_{m 0}+\lambda\right)^{2}+(1+\lambda)(1+z)^{3} \Omega_{m 0}\right]^{1 / 2}}
$$


and its current value is

$$
q_{0}=-1+\frac{\frac{3}{2} \Omega_{m 0}}{3\left(1-\Omega_{m 0}+\lambda\right)+(1+\lambda)^{-1}\left[\left(1-\Omega_{m 0}+\lambda\right)^{2}+(1+\lambda) \Omega_{m 0}\right]^{1 / 2}} .
$$

Finally, from relation (2.35) we can calculate the transition redshit as

$$
\begin{aligned}
& z_{t r}=-1+\frac{6^{1 / 3}(1+\lambda)^{-1 / 3} \Omega_{m 0}^{-1 / 3}}{3} \\
& \left\{10+9 \lambda\left(2+\lambda-\Omega_{m 0}\right)-9 \Omega_{m 0}\right. \\
& \left.-\sqrt{28-36 \Omega_{m 0}+9\left[3 \lambda(2+\lambda)-4 \lambda \Omega_{m 0}+\Omega_{m 0}^{2}\right]}\right\}^{1 / 3} .
\end{aligned}
$$

\section{Data and Methodology}

In this section we describe the various datasets that are going to be used in our analysis, and also the involved statistical methods. In particular, we will use data from direct measurements of the Hubble parameter, from Supernovae Type Ia (SNIa), and from Baryonic Acoustic Oscillations. Finally, we present various information criteria that offer information on the quality of the fit.

\subsection{Cosmological probes}

\subsubsection{Direct measurements of the Hubble expansion}

From the latest $H(z)$ data set compilation available in Ref. [64] we use only data obtained from cosmic chronometers (CC). By using the differential age of passive evolving galaxies one can measure the Hubble rate directly (see e.g. Ref. [66] and references therein). These galaxies are massive galaxies that evolve "slowly" at certain intervals of the cosmic time, i.e with small fraction of "new" stars. A striking advantage of the differential age of passive evolving galaxies is that the resulting measurement of the Hubble rate comes without any assumptions for the underlying cosmology, with the exception of imposed spatial flatness. Our study incorporates $N=31$ measurements of the Hubble expansion in the redshift range $0.07 \lesssim z \lesssim 2.0$.

Here, the corresponding $\chi_{H}^{2}$ function reads

$$
\chi_{H}^{2}\left(\phi^{v}\right)=\sum_{i=1}^{N} \frac{\left[H_{i}^{o b s}-H_{t h}\left(z_{i} ; \phi^{\nu}\right)\right]^{2}}{\sigma_{i}^{2}},
$$

where $H_{i}^{\text {obs }}$ is the observed Hubble rate at redshift $z_{i}$ and $\sigma_{i}$ the corresponding uncertainty, while $\phi^{v}$ is the statistical vector that contains the free parameters of the examined model.

\subsubsection{Supernovae Type Ia}

The most common class of cosmological probes is the so-called "standard" candles. The latter are luminous extra-galactic astrophysical objects with observable features that are independent of the cosmic time. The most known standard candles and probably the most thoroughly studied are Supernovae Type Ia (SNIa). In our analysis we use the most recent SNIa dataset available, i.e the binned Pantheon sample of Scolnic et. al. [67]. The full dataset is approximated very 
efficiently with the binned $N=40$ data points belonging to the redshift interval $0.01 \lesssim z \lesssim 1.6$. The corresponding $\chi^{2}$ is

$$
\chi_{\text {SNIa }}^{2}\left(\phi^{v+1}\right)=\mu_{\text {SNIa }} \mathbf{C}_{\text {SNIa,cov }}^{-1} \mu_{\text {SNIa }}^{T},
$$

where $\mu_{\mathrm{SNIa}}=\left\{\mu_{1}-\mu_{\mathrm{th}}\left(z_{1}, \phi^{\nu}\right), \ldots, \mu_{N}-\mu_{\mathrm{th}}\left(z_{N}, \phi^{\nu}\right)\right\}$. The distance modulus reads as $\mu_{i}=\mu_{B, i}-\mathcal{M}$, with $\mu_{B, i}$ the apparent maximum magnitude for redshift $z_{i}$. Here, $\mathcal{M}$ is a hyper-parameter [67] that quantifies uncertainties of various origins, such as astrophysical ones, data-reduction pipeline, etc, and it is employed instead of the usage of $\alpha, \beta$ free parameters, in the context of "BEAMS with Bias Corrections" method [65]. The observed distance modulus is compared with the theoretical one, i.e

$$
\mu_{\mathrm{th}}=5 \log \left(\frac{d_{L}\left(z ; \phi^{v}\right)}{\mathrm{Mpc}}\right)+25
$$

with

$$
d_{L}\left(z ; \phi^{v}\right)=c(1+z) \int_{0}^{z} \frac{d x}{H\left(x, \phi^{v}\right)}
$$

the luminosity distance for flat FRW geometry. It must be noted that $\mathcal{M}$ and the normalized Hubble constant $h$ are degenerate in light of Pantheon dataset in an intrinsic way, as it is usual in standard candles. Therefore, one should jointly employ other data-sets in order to obtain meaningful information regarding the present value $H_{0}$.

\subsubsection{Baryonic Acoustic Oscillations}

Baryonic Acoustic Oscillations refer to the imprint left by relativistic sound waves in the early universe, providing an observable to the late-time large scale structure. The main idea is to measure the aforementioned scale at different times (i.e redshifts), and thus obtain $D_{A}(z)$ and $H(z)$. The acoustic length scale corresponds to the co-moving distance that the sound waves could travel until the recombination $z_{*}[68]$, namely

$$
r_{d}=\int_{z_{*}}^{\infty} \frac{c_{s}(z)}{H(z)}
$$

For the concordance model, the sound speed, $c_{s}$, is given from an analytical expression. However, for the models considered here there is not such an expression, therefore the scale $r_{d}$ will be addressed as a free parameter. Furthermore, distances of different objects along the line of sight correspond to different redshifts and thus depend on the combination $H(z) r_{d}$, while distances transverse to the line of sight are related with the combination $D_{A}(z) / r_{d}$.

Employing large samples of tracers, (i.e galaxies), one can detect by statistical means the BAO peak, (for details see [69] and references therein). In order to achieve this it is required to impose an underlying cosmology, and hence the method is not model independent. However, the differences that may infiltrate at the final data products are much less than the statistical errors, and in most cases the data points are calibrated with the quantity $r_{d, f i d} / r_{d}$. In this work we employ the BAOs data-set used by [70], that consists of $N=11$ data points in the redshift range $0.106 \lesssim z \lesssim 2.36$. The relevant $\chi^{2}$ function reads as

$$
\chi_{B A O}^{2}\left(\phi^{v+1}\right)=\mathbf{s} C_{c o v}^{-1} \mathbf{s}^{T}+\sum_{i=8}^{N} \frac{\left[T\left(z_{i} ; \phi^{\nu}\right)-T_{i}^{o b s}\right]^{2}}{\sigma_{i}^{2}},
$$


with $C_{c o v}^{-1}$ the inverse of the covariance matrix of the first 6 measurements available at [70]. The vector $\mathbf{s}$ has as elements the $s_{i}$, given as $s_{i}=d_{m}-d_{i}^{o b s} r_{d} / r_{d, f i d}$ for odd i and $s_{i}=H\left(z_{i} ; \phi^{v}\right)-$ $H_{i}^{o b s} r_{d, f i d} / r_{d}$ for even i. In all cases, $r_{d, f i d}=147.78$. Furthermore, for $i \in\{8,9\}, T\left(z_{i} ; \phi^{v}\right)=$ $D_{v}\left(z_{i} ; \phi^{v}\right), T_{i}^{o b s}=D_{v, i}^{o b s} r_{d} / r_{d, f i d}$, with $r_{f i d, 8}=148.69 \mathrm{Mpc}$ and $r_{f i d, 9}=147.66 \mathrm{Mpc}$ respectively. For $i=10, T\left(z_{i} ; \phi^{\nu}\right)=c H\left(z_{i} ; \phi^{\nu}\right)^{-0.7} D_{m}\left(z_{i} ; \phi^{\nu}\right)^{0.3} / r_{d}$ and for $i=11, T\left(z_{i} ; \phi^{\nu}\right)=c H\left(z_{i} ; \phi^{\nu}\right)^{-1} r_{d}$. Finally, in the expressions above the following quantities have been used

$$
\begin{gathered}
D_{M}\left(z_{i} ; \phi^{v}\right)=\frac{D_{L}\left(z_{i} ; \phi^{v}\right)}{1+z}, \\
D_{A}\left(z_{i} ; \phi^{v}\right)=\frac{D_{L}\left(z_{i} ; \phi^{v}\right)}{(1+z)^{2}} \\
D_{V}\left(z ; \phi^{v}\right)=\left[\frac{c D_{A}\left(z ; \phi^{v}\right)^{2} z(1+z)^{2}}{H\left(z ; \phi^{v}\right)}\right]^{1 / 3} .
\end{gathered}
$$

\subsubsection{Big Bang Nucleosynthesis}

Any cosmological scenario arising from modified gravity should preserve the standard thermal history of the universe. Hence, a basic and rough condition is applicable in the form of an extra prior. Specifically, we require that the following inequality holds [71-73]

$$
\frac{\left(H_{i}\left(z_{B B N} ; \phi^{v}\right)-H_{\Lambda}\left(z_{B B N} ; \Omega_{m 0}\right)\right)^{2}}{H_{\Lambda}\left(z_{B B N} ; \Omega_{m 0}\right)^{2}}<0.1,
$$

where $z_{B B N} \sim 10^{9}$. For the fiducial $\Lambda \mathrm{CDM}$ cosmology, namely $H_{\Lambda}$, we employ the parameter values from Planck [74].

\subsubsection{Joint likelihood analysis}

In order to obtain the joint constraints on the cosmological parameters from the aforementioned cosmological probes, we introduce the total likelihood function as

$$
\mathcal{L}_{\text {tot }}\left(\phi^{k}\right)=\mathcal{L}_{S N I a} \times \mathcal{L}_{H} \times \mathcal{L}_{B A O} .
$$

It is easy to deduce that relevant $\chi_{\text {tot }}^{2}$ is given as

$$
\chi_{\mathrm{tot}}^{2}\left(\phi^{k}\right)=\chi_{S N I a}^{2}+\chi_{H}^{2}+\chi_{B A O}^{2}
$$

The involved statistical vector has $k$ components, i.e. the $v$ parameters of the scenario at hand plus $v_{\text {hyp }}$ hyper-parameters from the imposed datasets, namely $k=v+v_{\text {hyp }}$. Hence, the vector containing the free parameters of the scenaria at hand is $\phi^{k}=\left\{\Omega_{m 0}, h, \lambda, \mathcal{M}, r_{d}\right\}$. Note however that from a statistical point of view there is no distinction between the intrinsic hyper-parameters of a given dataset and the free parameters of a cosmological scenario.

Finally, for the likelihood maximization we use an affine-invariant Markov Chain Monte Carlo sampler [75], obtained in the Python package emcee [76]. We use 1000 chains (walkers) and 3500 steps (states). As a prior we employ firstly the conditions $0.0<\Omega_{m 0}<1,0.60<h<0.90$, $-19.9<M<-18.0,-0.9<\lambda<2.8,135<r_{d}<160$, and secondly the BBN constraint described above. Lastly, the convergence of the MCMC algorithm is verified with auto-correlation time implementation, and moreover for completeness the Gelman-Rubin criterion is calculated. 


\subsection{Information Criteria and Model Selection}

As a last step we present the standard ways in order to compare a set of cosmological scenarios, namely we apply the Akaike Information Criterion (AIC) [77], the Bayesian Information Criterion (BIC) [78], and the Deviance Information Criterion [79]. Moreover, we present the standard $\chi_{\min }^{2} /$ dof, where "dof" stands for degrees of freedom, usually defined as the number of the used data points minus the number of fitted parameters. In our case this gives dof $=77$. Nevertheless, $\chi_{\min }^{2} /$ dof should be used for illustrative purposes, as the degrees of freedom might be ambiguous for non-linear (in terms of the free parameters) models. [80].

The AIC criterion is based on information theory and it is an asymptotically unbiased estimator of the Kullback-Leibler information. Under the standard assumption of Gaussian errors, the corresponding estimator for the AIC criterion reads [81, 82]

$$
\mathrm{AIC}=-2 \ln \left(\mathcal{L}_{\max }\right)+2 k+\frac{2 k(k+1)}{N_{\mathrm{tot}}-k-1},
$$

with $\mathcal{L}_{\max }$ the maximum likelihood of the dataset(s) under consideration and $N_{\text {tot }}$ the total data points number. It is apparent that for $N_{\text {tot }}>>1$ this expression gives the original AIC version, namely $\mathrm{AIC} \simeq-2 \ln \left(\mathcal{L}_{\max }\right)+2 k$. As it is discussed in [83], it is considered as best practise to use the modified AIC criterion.

The BIC criterion is a Bayesian evidence estimator, and it is written as [81-83]

$$
\mathrm{BIC}=-2 \ln \left(\mathcal{L}_{\max }\right)+k \log \left(N_{\text {tot }}\right) .
$$

Finally, the DIC criterion employs both Bayesian statistics and information theory concepts [79], and it is expressed as [83]

$$
\mathrm{DIC}=D\left(\overline{\phi^{k}}\right)+2 C_{B} .
$$

The quantity $C_{B}$ is the Bayesian complexity $C_{B}=\overline{D\left(\phi^{k}\right)}-D\left(\overline{\phi^{k}}\right)$, where overlines imply the standard mean value. Moreover, $D\left(\phi^{k}\right)$ is the Bayesian Deviation, which can be expressed as $D\left(\phi^{k}\right)=-2 \ln \left[\mathcal{L}\left(\phi^{k}\right)\right]$ in the case of exponential class of distributions. It is closely related to the number of effective degrees of freedom [79], which is actually the number of parameters that affect the fitting. In a less strict manner, it could be considered as a measure of the "spread" of the likelihood.

In contrast with AIC and BIC criteria, instead of using just the best fit likelihood, DIC uses the whole sample. Furthermore, AIC and BIC count and penalize all the involved parameters, while DIC penalizes only the number of parameters that contribute to the fit in an actual way. Finally, an additional appealing feature of DIC criterion is that its calculation is computationally light under the MCMC samples.

Given a set of scenarios that describe the same class of phenomena, our problem is to sort the models according to their fitting efficiency in the context of the available data. We employ the aforementioned three information criteria (IC) and we calculate the relative difference of the IC value for the given set of models, $\Delta \mathrm{IC}_{\text {model }}=\mathrm{IC}_{\text {model }}-\mathrm{IC}_{\mathrm{min}}$, where the $\mathrm{IC}_{\min }$ is the minimum IC value inside the competing models set. In order to qualify each model in terms of its relevant adequacy, we apply the Jeffreys scale [84]. Specifically, the condition $\Delta \mathrm{IC} \leq 2$ implies statistical 


\begin{tabular}{cccccccc}
\hline \hline Model & $\Omega_{m 0}$ & $h$ & $\lambda$ & $r_{d}$ & $\mathcal{M}$ & $\chi_{\min }^{2}$ & $\chi_{\min }^{2} /$ dof \\
\hline \hline Mod. 1 & $0.425_{-0.146}^{+0.107}$ & $0.691_{-0.017}^{+0.016}$ & $0.491_{-0.533}^{+0.387}$ & $146.20_{-3.41}^{+2.55}$ & $-19.382_{-0.052}^{+0.051}$ & 61.93 & 0.8043 \\
Mod. 2 & $0.339_{-0.122}^{+0.093}$ & $0.679_{-0.016}^{+0.016}$ & $0.537_{-0.550}^{+0.403}$ & $146.60_{-3.44}^{+3.57}$ & $-19.396_{-0.052}^{+0.051}$ & 63.53 & 0.8251 \\
$\Lambda$ CDM & $0.292_{-0.014}^{+0.015}$ & $0.692_{-0.017}^{+0.017}$ & - & $145.87_{-3.38}^{+3.53}$ & $-19.377_{-0.052}^{+0.051}$ & 61.73 & 0.7914 \\
\hline \hline
\end{tabular}

Table 1. Observational constraints and the corresponding $\chi_{\min }^{2}$, as well as $\chi_{\min }^{2} /$ dof (where "dof" stands for degrees of freedom, in our case dof $=77$ ), for the two Myrzakulov gravity models, presented previously, using CC/Pantheon/BAO data-sets. In order to allow direct comparison, the concordance flat $\Lambda$ CDM model is also analyzed, giving results very similar with the corresponding ones of [85].

compatibility with the most favoured model by the data, while the condition $2<\Delta \mathrm{IC}<6$ corresponds to middle tension between the two models, and lastly the condition $\Delta \mathrm{IC} \geq 10$ implies strong tension.

\section{Results}

In this section we proceed to the observational analysis of Myrzakulov gravity using the datasets and the methods described above. Note that the free parameters of the aforementioned models are $\Omega_{m 0}, h$ and $\lambda$, while for the case of the concordance cosmology they are $\Omega_{m 0}, h$. For convenience we summarize the obtained results in Table 1. Additionally, in Figs. 1 and 2 we present the corresponding contour plots for Model 1 and Model 2 respectively. For comparison and benchmark we also analyzed the concordance model, namely $\Lambda \mathrm{CDM}$.

As we can see, according to the combined analysis of $\mathrm{CC}+\mathrm{SNIa}+\mathrm{BAO}$ data we acquire $\sim 1 \sigma$ compatibility in all cases. The dimensionless parameter $\lambda$ is constrained to an interval around 0 , that includes $\Lambda \mathrm{CDM}$ paradigm, which was expected since as we discussed above a realistic modified gravity should be a small deviation from general relativity. Nevertheless, note that in both Model 1 and Model 2, the $\lambda$-contours are slightly shifted towards positive values. We mention that having the likelihood contours for the parameter $\lambda$ allows us to extract the constraints on the parameter $c$ through expression (2.25) for Model 1 and on the parameter $c_{1}$ through (2.34) for Model 2. In particular, for $1 \sigma$ region for Model 1 we obtain $c=1.550_{-0.876}^{+0.828}$, while for Model 2 we find $c_{1}=4.94_{-2.75}^{+2.28}$.

Concerning the values of $\Omega_{m 0}$ we observe that Model 1 gives a rather large value, due to the degeneracy with $\lambda$, while for Model 2 this is not the case. Concerning the Hubble constant $h$, for Model 1 we find that $0.690_{-0.017}^{+0.016}$, while for Model 2 we obtain $0.679_{-0.016}^{+0.016}$. This implies that the obtained values for the present Hubble parameter $H_{0}$ are in between the Planck estimation $H_{0}=67.36 \pm 0.54 \mathrm{~km} / \mathrm{s} / \mathrm{Mpc}$ [74] and the local estimation $H_{0}=73.24 \pm 1.74 \mathrm{~km} / \mathrm{s} / \mathrm{Mpc}$ [86], although closer to the former. In addition, the extracted $H_{0}$ value for both models is consistent with other astrophysical inferences of Hubble constant, i.e $H_{0}=67.4_{-3.2}^{+4.1} \mathrm{kms}^{-1} \mathrm{Mpc}^{-1}$ [87], and $H_{0}=69.6 \pm 2.5 \mathrm{kms}^{-1} \mathrm{Mpc}^{-1}$ [88]. It is interesting to note that results in this range have been supported for about a decade now, amongst others in [89-95].

In order to provide a more complete and transparent picture, we use the obtained allowed parameter values in order to extract the resulting $H(z)$. In Figs. 3 and 4 we present the reconstructed mean $H(z) /(z+1)$ as a function of the redshift, alongside the allowed curves for the $1 \sigma$ allowed 


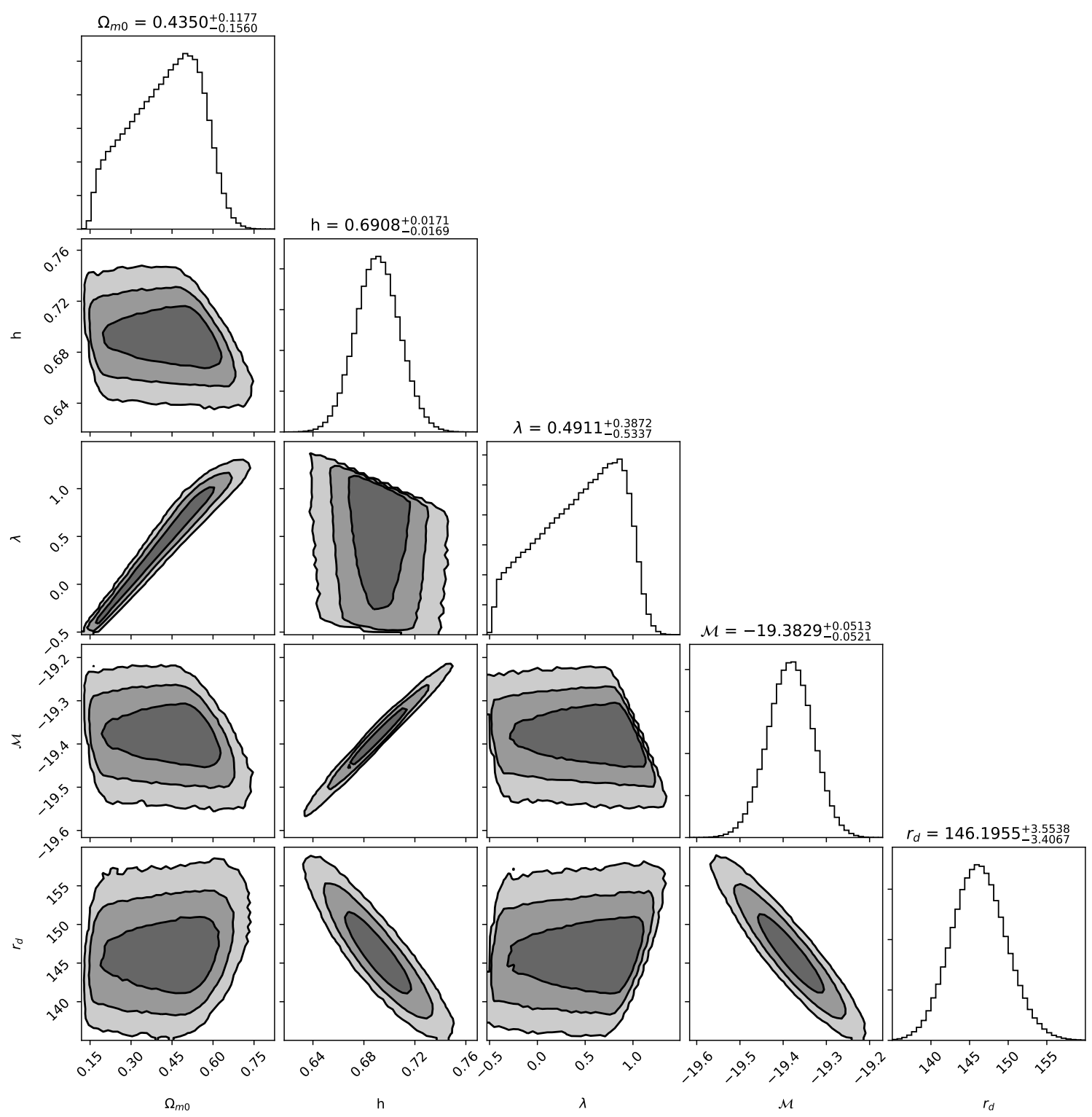

Figure 1. The $1 \sigma, 2 \sigma$ and $3 \sigma$ likelihood contours for Model 1 of (2.22),(2.23), for all possible $2 D$ subsets of the parameter space $\left(\Omega_{m 0}, h, \lambda, \mathcal{M}, r_{d}\right)$. Moreover, we present the mean parameter values within the $1 \sigma$ area of the MCMC chain. We have performed a joint analysis of CC+SNIa+BAO data.

model parameters presented above, for Model 1 and Model 2 respectively. These graphs are quite similar with the corresponding ones for quintessence models $(\phi C D M)$ of [96].

As a next step we investigate of the evolution of the dark energy equation-of-state parameter. In particular, having obtained the allowed parameter values at $1 \sigma$ confidence level, we can use them in order to extract the resulting $w_{d e}(z)$ behavior given by (2.19), with the deceleration parameter 


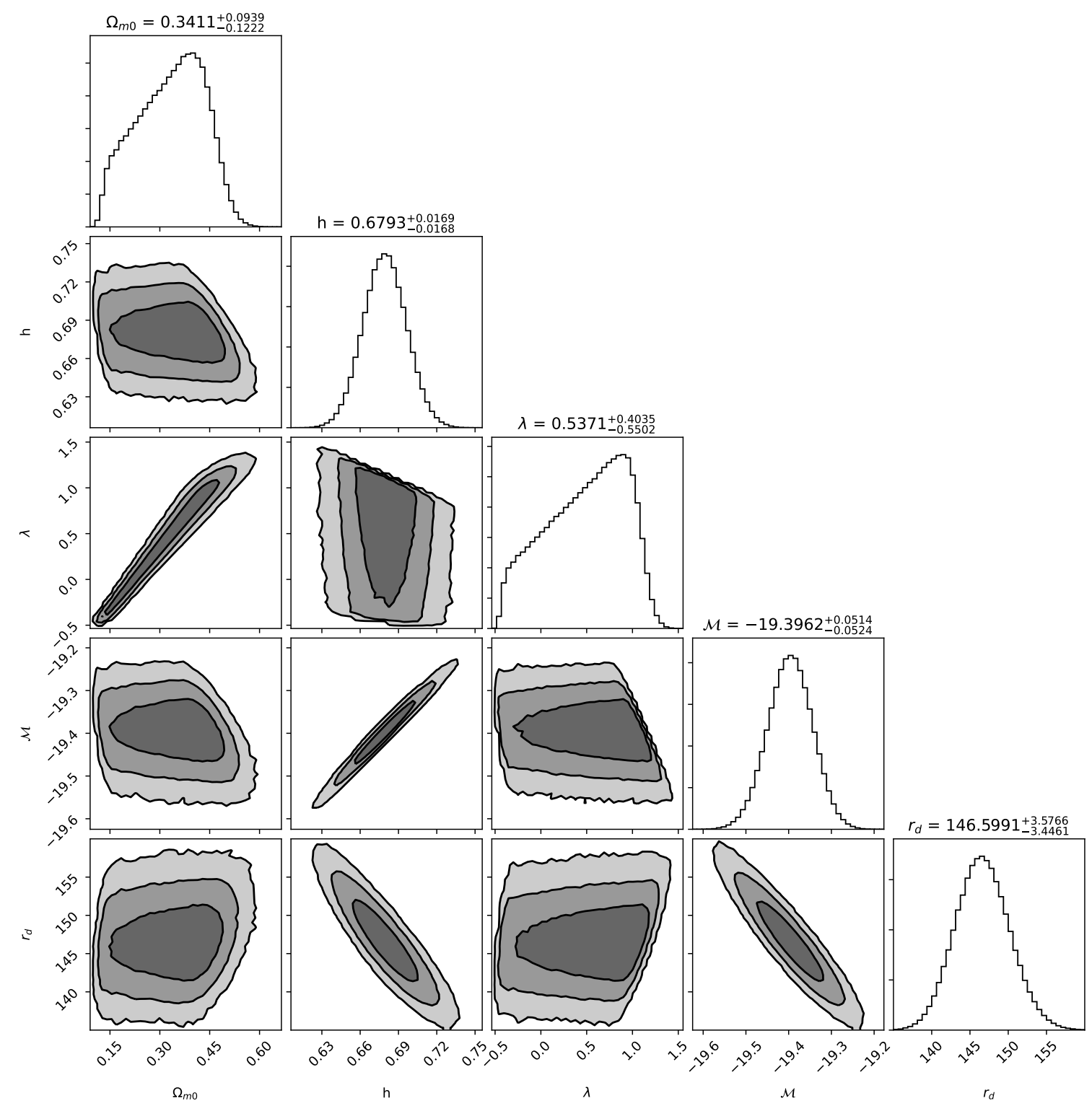

Figure 2. The $1 \sigma, 2 \sigma$ and $3 \sigma$ likelihood contours for Model 2 of (2.31),(2.32), for all possible 2D subsets of the parameter space $\left(\Omega_{m 0}, h, \lambda, \mathcal{M}, r_{d}\right)$. Moreover, we present the mean parameter values within the $1 \sigma$ area of the MCMC chain. We have performed a joint analysis of CC+SNIa+BAO data.

given by (2.26) for Model 1 and by (2.35) for Model 2.

In Fig. 5 we depict the reconstructed mean $w_{d e}(z)$ (red curve) for Model 1, alongside the allowed curves for the $1 \sigma$ allowed model parameters presented above. As we observe, the corresponding behavior is very close to $\Lambda$ CDM scenario for every parameter values. Similarly, in Fig. 6 we present the corresponding graph for Model 2. In this case the scenario resembles $\Lambda$ CDM at low redshifts, however at earlier times the mean curve, as well as many of the "individual" 


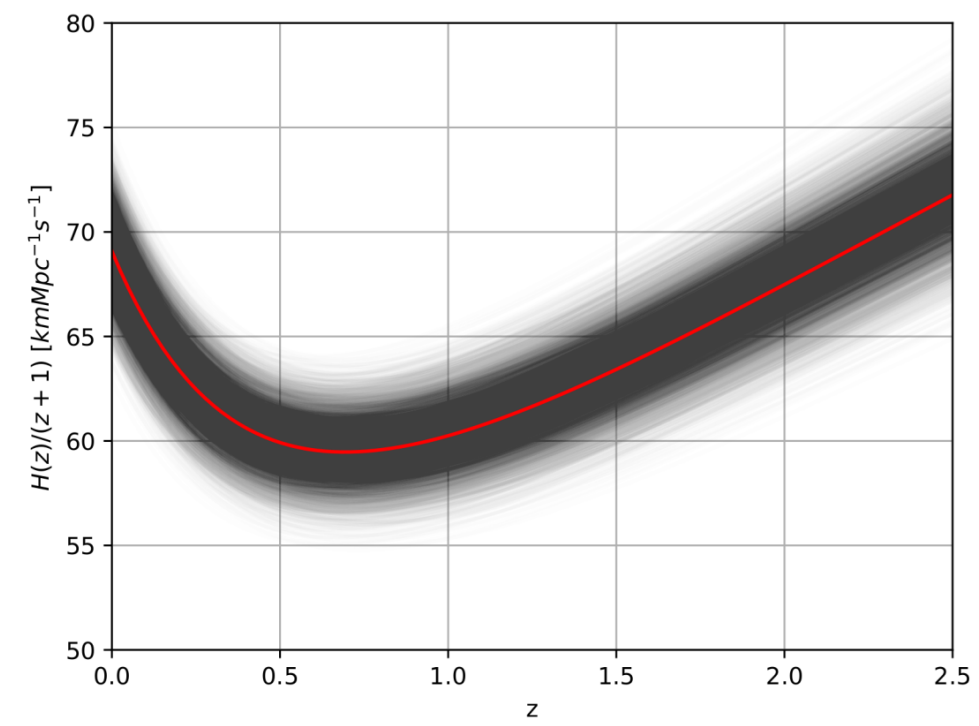

Figure 3. The reconstruction of the $H(z) /(z+1)$ as a function of the redshift for Model 1 , arisen from (2.20),(2.22). We re-sampled the chains produced by emcee taking 6000 samples, and we plot all the obtained curves, alongside the curve corresponding to the best fit of the parameters (red curve).

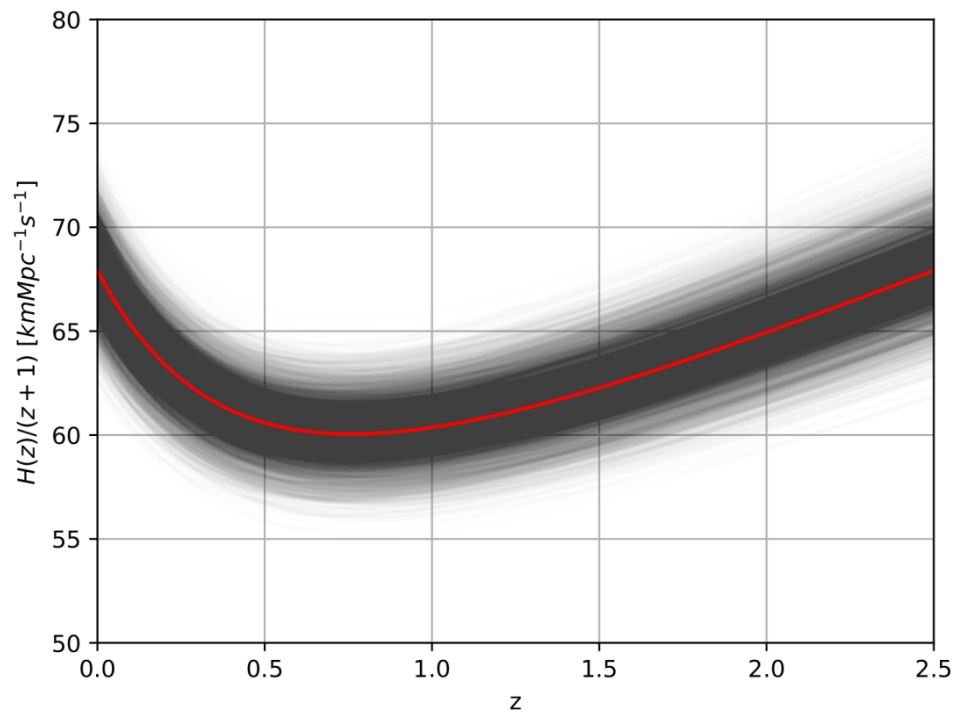

Figure 4. The reconstruction of the $H(z) /(z+1)$ as a function of the redshift for Model 2, arisen from (2.29),(2.31). We re-sampled the chains produced by emcee taking 6000 samples, and we plot all the obtained curves, alongside the curve corresponding to the best fit of the parameters (red curve). 


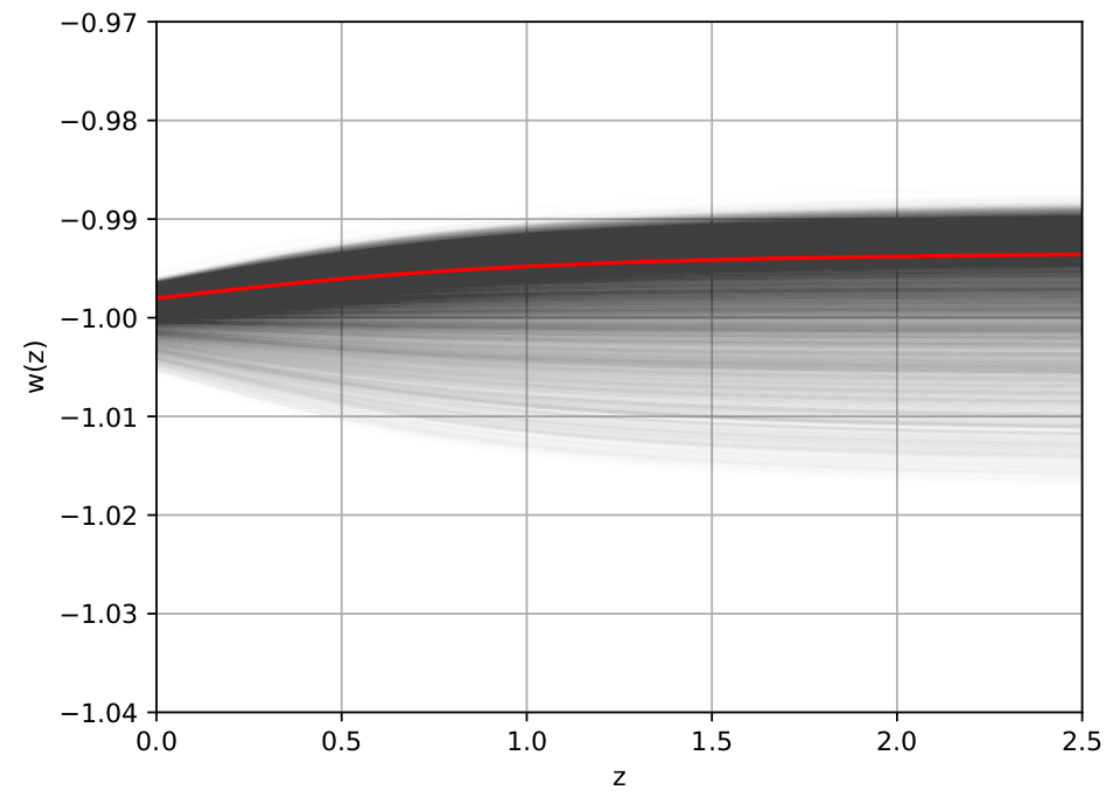

Figure 5. The reconstruction of the effective dark-energy equation-of-state parameter $w_{d e}(z)$ as a function of the redshift for Model 1 given by (2.24). We re-sampled the chains produced by emcee taking 6000 samples, and we plot all the obtained $w_{d e}(z)$ curves, alongside the curve corresponding to the best fit of the parameters (red curve).

curves, present a deviation, since this is allowed by the used datasets. In particular, for some parameter choices the dark-energy pressure at a particular redshift diverges and changes sign, and thus the $w_{d e}(z)$ transits on the other side of the phantom-divide. Such energy conditions violations are common in modified gravity theories, and actually they can lead to interesting cosmological phenomenology. Note that the observable quantities (the Hubble function and its derivatives, the density parameters etc) remain finite. However, we mention that a significant sub-set of the curves, i.e. a large region of the parameter space of the model, does not exhibit such a behavior, and the individual obtained curves resemble the $\Lambda \mathrm{CDM}$ evolution.

Furthermore, we proceed to the reconstruction of the decceleration parameter using random sampling of the obtained chains. Concerning the current value $q_{0}$, for Model 1 using (2.27) we obtain $q_{0}=-0.561_{-0.021}^{+0.022}$, while for Model 2 using (2.36) we acquire $q_{0}=-0.880_{-0.009}^{+0.010}$. These are in agreement with the values obtained using other datasets, such as supernovae, quasars and gamma-ray bursts by means of model-independent techniques [97].

Additionally, we calculate the transition redshift, for the two models, using relations (2.28) and (2.37) respectively. For Model 1 we find $z_{\mathrm{tr}, 1}=0.36_{-0.18}^{+0.10}$, while for Model 2 we acquire $z_{\mathrm{tr}, 2}=0.74_{-0.14}^{+0.07}$. It is of interest to compare the aforementioned values with $z_{\mathrm{tr}, A}=0.72 \pm 0.05$, [96] and $z_{\mathrm{tr}, B}=0.64_{-0.09}^{+0.12}$, [98]. For Model 1 we observe mild compatibility within $\sim 3.5 \sigma$ and within $\sim 3 \sigma$ with "A" and "B" results, respectively. On the other hand, for the case of Model 2 we 


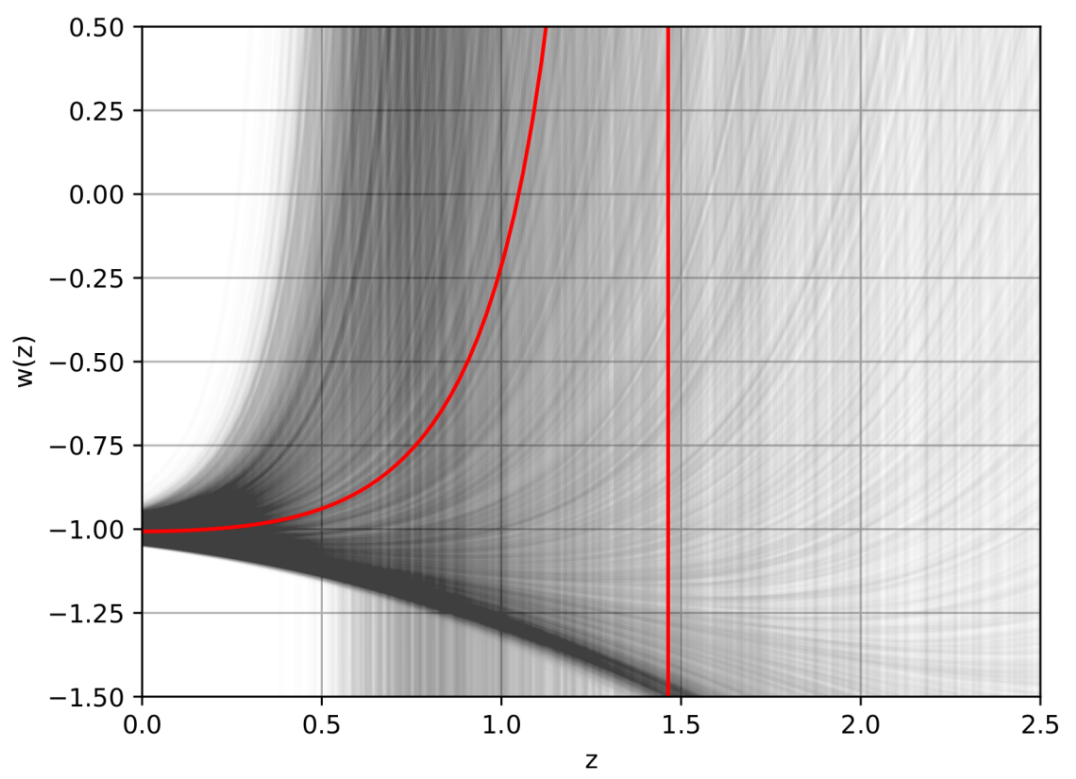

Figure 6. The reconstruction of the effective dark-energy equation-of-state parameter $w_{d e}(z)$ as a function of the redshift for Model 2 given by (2.33). We re-sampled the chains produced by emcee taking 6000 samples, and we plot all the obtained $w_{d e}(z)$ curves, alongside the curve corresponding to the best fit of the parameters (red curve). The over-populated area at the bottom corresponds to a peak within $1 \sigma$ area, nevertheless since we extract the median value of each parameter within $1 \sigma$ as the best fit, the "best" $w_{d e}(z)$ curve differs.

report $1 \sigma$ compatibility with both results. These results act as an additional verification check of the examined models.

We close this analysis with the examination of the statistical significance of our fitting results, applying the AIC, BIC and DIC information criteria described in subsection 3.2. We summarize our results in Table 2. As we observe, Model 1 is statistically equivalent with $\Lambda \mathrm{CDM}$ paradigm, and especially the combined and more complete DIC criterion gives an almost equal value. Additionally, Model 2 also presents a very good fitting behavior, and according to DIC it is also statistically equivalent with $\Lambda \mathrm{CDM}$ paradigm, which is an interesting result since Model 2 does not contain $\Lambda \mathrm{CDM}$ scenario as a limit for any parameter value.

\section{Conclusions}

In this work we have used observational data from Supernovae (SNIa) Pantheon sample, from Baryonic Acoustic Oscillations (BAO), and from cosmic chronometers measurements of the Hubble parameter (CC), alongside arguments from Big Bang Nucleosynthesis (BBN), in order to extract constraints on Myrzakulov $F(R, T)$ gravity. This is a connection-based theory belonging to the Riemann-Cartan subclass, that uses a specific but non-special connection, which then leads to extra 


\begin{tabular}{ccccccc}
\hline \hline Model & AIC & $\Delta$ AIC & BIC & $\Delta$ BIC & DIC & $\Delta$ DIC \\
\hline \hline Mod. 1 & 72.7234 & 2.4757 & 83.9675 & 4.6124 & 69.6728 & 0.0007 \\
Mod. 2 & 74.3204 & 4.0727 & 85.5645 & 6.2094 & 71.3725 & 1.7004 \\
$\Lambda$ CDM & 70.2477 & 0 & 79.3551 & 0 & 69.6721 & 0 \\
\hline \hline
\end{tabular}

Table 2. The information criteria AIC, BIC and DIC for the examined cosmological models, alongside the relative difference from the best-fitted model $\Delta \mathrm{IC} \equiv \mathrm{IC}-\mathrm{IC}_{\min }$.

degrees of freedom. One introduces a parametrization that quantifies the deviation of torsion and curvature scalars form their values corresponding to the special Levi-Civita and Weitzenböck connections, and then constructs various models by assuming specific forms for the involved functions. In all models, one obtains an effective dark-energy sector of geometrical origin.

We considered two specific models, which are known to lead to interesting phenomenology. Our analysis shows that both models are capable of describing adequately the imposed datasets, namely $\mathrm{CC}+\mathrm{SNIa}+\mathrm{BAO}$ ones, obtaining $\sim 1 \sigma$ compatibility in all cases. Concerning Model 1 , which includes $\Lambda \mathrm{CDM}$ paradigm as a particular limit, we found a relatively large value for $\Omega_{m 0}$ and a value for $h$ in between the Planck and local estimation, although closer to the former. For the dimensionless parameter $\lambda$ we found that it is constrained to an interval around 0 , which corresponds to $\Lambda \mathrm{CDM}$ scenario, however the corresponding contours are slightly shifted towards positive values. In the case of Model 2, we found smaller $\Omega_{m 0}$ and $h$, while $\lambda$ is again constrained around 0 with favoured positive values.

Additionally, we used the obtained posterior distribution of the parameters at $1 \sigma$ confidence level, and we reconstructed the Hubble function as a function of the redshift. As we showed, the obtained graphs for $H(z) /(z+1)$ are in very good agreement with observations. Furthermore, we reconstructed the induced dark-energy equation-of-state parameter as a function of the redshift. As we saw, for Model $1 w_{d e}(z)$ is very close to $\Lambda$ CDM scenario, while for Model 2 it resembles $\Lambda$ CDM at low redshifts, however at earlier times deviations are allowed.

Finally, applying the AIC, BIC and the combined DIC criteria, we deduced that both Model 1 and Model 2 present a very efficient fitting behavior, and are statistically equivalent with $\Lambda$ CDM cosmology. This is an interesting result since Model 2 does not contain $\Lambda$ CDM scenario as a limit for any parameter value.

In summary, Myrzakulov $F(R, T)$ gravity is in agreement with cosmological data, and it could serve as a candidate for the description of nature. Nevertheless, one should also investigate the theory at the perturbation level and confront it with perturbation-related data, i.e $f \sigma_{8}$. Such an analysis, although both interesting and necessary, lies beyond the scope of the present work and it is left for a future project.

\section{References}

[1] K. A. Olive, Inflation, Phys. Rept. 190, 307 (1990).

[2] N. Bartolo, E. Komatsu, S. Matarrese and A. Riotto, Non-Gaussianity from inflation: Theory and observations, Phys. Rept. 402, 103 (2004) [arXiv: astro-ph/ 0406398 ]. 
[3] E. J. Copeland, M. Sami and S. Tsujikawa, Dynamics of dark energy, Int. J. Mod. Phys. D 15, 1753 (2006) [arXiv:hep-th/0603057].

[4] Y. -F. Cai, E. N. Saridakis, M. R. Setare and J. -Q. Xia, Quintom Cosmology: Theoretical implications and observations, Phys. Rept. 493, 1 (2010) [arXiv: 0909 . 2776].

[5] S. Capozziello and M. De Laurentis, Extended Theories of Gravity, Phys. Rept. 509, 167 (2011) [arXiv:1108.6266].

[6] Y. F. Cai, S. Capozziello, M. De Laurentis and E. N. Saridakis, $f(T)$ teleparallel gravity and cosmology, Rept. Prog. Phys. 79, 106901 (2016) [arXiv: 1511.07586 ].

[7] P. Brax, C. van de Bruck and A. C. Davis, Brane world cosmology, Rept. Prog. Phys. 67, 2183-2232 (2004) [arXiv: hep-th/0404011].

[8] A. De Felice and S. Tsujikawa, $f(R)$ theories, Living Rev. Rel. 13, 3 (2010) [arXiv: 1002 . 4928 ].

[9] S. Nojiri and S. D. Odintsov, Unified cosmic history in modified gravity: from $F(R)$ theory to Lorentz non-invariant models, Phys. Rept. 505, 59 (2011) [arXiv: 1011.054 4].

[10] S. Nojiri and S. D. Odintsov, Modified Gauss-Bonnet theory as gravitational alternative for dark energy, Phys. Lett. B 631, 1 (2005) [arXiv: hep-th/ 0508049 ].

[11] A. De Felice and S. Tsujikawa, Construction of cosmologically viable $f(G)$ dark energy models, Phys. Lett. B 675, 1 (2009) [arXiv:0810 . 5712].

[12] D. Lovelock, The Einstein tensor and its generalizations, J. Math. Phys. 12, 498 (1971).

[13] N. Deruelle and L. Farina-Busto, The Lovelock Gravitational Field Equations in Cosmology, Phys. Rev. D 41, 3696 (1990).

[14] G. W. Horndeski, Second-order scalar-tensor field equations in a four-dimensional space, Int. J. Theor. Phys. 10, 363-384 (1974).

[15] A. Nicolis, R. Rattazzi and E. Trincherini, The Galileon as a local modification of gravity, Phys. Rev. D 79, 064036 (2009) [arXiv: 0811 .2197].

[16] C. Deffayet, G. Esposito-Farese and A. Vikman, Covariant Galileon, Phys. Rev. D 79, 084003 (2009) [arXiv:0901.1314].

[17] R. Aldrovandi and J. G. Pereira, Teleparallel Gravity: An Introduction, Springer, Dordrecht (2013).

[18] J. W. Maluf, The teleparallel equivalent of general relativity, Annalen Phys. 525, (2013) 339, [arXiv:1303.3897].

[19] R. Ferraro and F. Fiorini, Modified teleparallel gravity: Inflation without inflaton, Phys. Rev. D 75, 084031 (2007) [arXiv:gr-qc/ 0610067 ].

[20] E. V. Linder, Einstein's Other Gravity and the Acceleration of the Universe, Phys. Rev. D 81 (2010) 127301, [arXiv: 1005.3039$]$.

[21] G. Kofinas and E. N. Saridakis, Teleparallel equivalent of Gauss-Bonnet gravity and its modifications, Phys. Rev. D 90, 084044 (2014) [arXiv: 1404 .2249].

[22] C.-Q. Geng, C.-C. Lee, E. N. Saridakis and Y.-P. Wu, Teleparallel dark energy, Phys. Lett. B 704 (2011) 384-387, [arXiv:1109.1092].

[23] M. Hohmann, L. Järv and U. Ualikhanova, Covariant formulation of scalar-torsion gravity, Phys. Rev. D 97, no.10, 104011 (2018) [arXiv: 1801.05786$].$

[24] F. W. Hehl, J. D. McCrea, E. W. Mielke and Y. Ne'eman, Metric affine gauge theory of gravity: 
Field equations, Noether identities, world spinors, and breaking of dilation invariance, Phys. Rept. 258, 1 (1995) [arXiv: gr-qc/9402012].

[25] J. Beltran Jimenez, A. Golovnev, M. Karciauskas and T. S. Koivisto, The Bimetric variational principle for General Relativity, Phys. Rev. D 86, 084024 (2012) [arXiv: 1201.4018 ].

[26] N. Tamanini, Variational approach to gravitational theories with two independent connections, Phys. Rev. D 86, 024004 (2012) [arXiv: 1205.2511].

[27] G. Y. Bogoslovsky and H. F. Goenner, Finslerian spaces possessing local relativistic symmetry, Gen. Rel. Grav. 31, 1565 (1999) [arXiv: gr-qc/ 9904081 ].

[28] N. E. Mavromatos, S. Sarkar and A. Vergou, Stringy Space-Time Foam, Finsler-like Metrics and Dark Matter Relics, Phys. Lett. B 696, 300 (2011) [arXiv: 1009.2880].

[29] S. Basilakos, A. P. Kouretsis, E. N. Saridakis and P. Stavrinos, Resembling dark energy and modified gravity with Finsler-Randers cosmology, Phys. Rev. D 88, 123510 (2013) [arXiv:1311. 5915].

[30] A. P. Kouretsis, M. Stathakopoulos and P. C. Stavrinos, Covariant kinematics and gravitational bounce in Finsler space-times, Phys. Rev. D 86, 124025 (2012) [arXiv: 1208 . 1673].

[31] A. Triantafyllopoulos and P. C. Stavrinos, Weak field equations and generalized FRW cosmology on the tangent Lorentz bundle, Class. Quant. Grav. 35, no. 8, 085011 (2018).

[32] S. Ikeda, E. N. Saridakis, P. C. Stavrinos and A. Triantafyllopoulos, Cosmology of Lorentz fiber-bundle induced scalar-tensor theories, Phys. Rev. D 100, no.12, 124035 (2019) [arXiv:1907.10950].

[33] A. Conroy and T. Koivisto, The spectrum of symmetric teleparallel gravity, Eur. Phys. J. C 78, no. 11, 923 (2018) [arXiv: 1710 . 05708].

[34] R. Myrzakulov, FRW Cosmology in F(R,T) gravity, Eur. Phys. J. C 72, 2203 (2012) [arXiv:1207.1039].

[35] E. N. Saridakis, S. Myrzakul, K. Myrzakulov and K. Yerzhanov, Cosmological applications of $F(R, T)$ gravity with dynamical curvature and torsion, Phys. Rev. D 102, no.2, 023525 (2020) [arXiv:1912.03882].

[36] M. Jamil, D. Momeni, M. Raza and R. Myrzakulov, Reconstruction of some cosmological models in $f(R, T)$ gravity, Eur. Phys. J. C 72, 1999 (2012) [arXiv:1107. 5807].

[37] M. Sharif, S. Rani and R. Myrzakulov, Analysis of $F(R, T)$ gravity models through energy conditions, Eur. Phys. J. Plus 128, 123 (2013) [arXiv: 1210 .2714].

[38] S. Capozziello, M. De Laurentis and R. Myrzakulov, Noether Symmetry Approach for teleparallel-curvature cosmology, Int. J. Geom. Meth. Mod. Phys. 12, no. 09, 1550095 (2015) [arXiv:1412.1471].

[39] P. Feola, X. J. Forteza, S. Capozziello, R. Cianci and S. Vignolo, The mass-radius relation for neutron stars in $f(R)=R+\alpha R^{2}$ gravity: a comparison between purely metric and torsion formulations, [arXiv:1909.08847].

[40] B. Feng, X. L. Wang and X. M. Zhang, Dark energy constraints from the cosmic age and supernova, Phys. Lett. B 607, 35-41 (2005) [arXiv:astro-ph/ 0404224 ].

[41] G. Olivares, F. Atrio-Barandela and D. Pavon, Observational constraints on interacting quintessence models, Phys. Rev. D 71, 063523 (2005) [arXiv: astro-ph/0503242].

[42] S. Capozziello, V. F. Cardone, E. Elizalde, S. Nojiri and S. D. Odintsov, Observational constraints 
on dark energy with generalized equations of state, Phys. Rev. D 73, 043512 (2006)

[arXiv:astro-ph/0508350].

[43] R. Maartens and E. Majerotto, Observational constraints on self-accelerating cosmology, Phys. Rev. D 74, 023004 (2006) [arXiv: astro-ph/ 0603353 ].

[44] R. Lazkoz, R. Maartens and E. Majerotto, Observational constraints on phantom-like braneworld cosmologies, Phys. Rev. D 74, 083510 (2006) [arXiv: astro-ph/ 0605701 ].

[45] W. M. Wood-Vasey et al. [ESSENCE], Observational Constraints on the Nature of the Dark Energy: First Cosmological Results from the ESSENCE Supernova Survey, Astrophys. J. 666, 694-715 (2007) [arXiv: astro-ph/ 0701041 ].

[46] X. Zhang and F. Q. Wu, Constraints on Holographic Dark Energy from Latest Supernovae, Galaxy Clustering, and Cosmic Microwave Background Anisotropy Observations, Phys. Rev. D 76, 023502 (2007) [arXiv:astro-ph/0701405].

[47] S. Tsujikawa, Observational signatures of $f(R)$ dark energy models that satisfy cosmological and local gravity constraints, Phys. Rev. D 77, 023507 (2008) [arXiv: 0709.1391].

[48] S. Basilakos, M. Plionis and J. Solà, Hubble expansion $\backslash \&$ Structure Formation in Time Varying Vacuum Models, Phys. Rev. D 80, 083511 (2009) [arXiv: 0907 . 4555].

[49] S. Dutta and E. N. Saridakis, Overall observational constraints on the running parameter $\lambda$ of Horava-Lifshitz gravity, JCAP 05, 013 (2010) [arXiv: 1002 . 3373].

[50] S. Nesseris, A. De Felice and S. Tsujikawa, Observational constraints on Galileon cosmology, Phys. Rev. D 82, 124054 (2010) [arXiv: 1010 . 0407].

[51] C. Q. Geng, C. C. Lee and E. N. Saridakis, Observational Constraints on Teleparallel Dark Energy, JCAP 01, 002 (2012) [arXiv: 1110 .0913].

[52] S. Basilakos, S. Nesseris and L. Perivolaropoulos, Observational constraints on viable $f(R)$ parametrizations with geometrical and dynamical probes, Phys. Rev. D 87, no.12, 123529 (2013) [arXiv:1302.6051].

[53] S. Basilakos and J. Solà, Growth index of matter perturbations in running vacuum models, Phys. Rev. D 92, no.12, 123501 (2015) [arXiv:1509.06732].

[54] W. Yang, S. Pan, E. Di Valentino, E. N. Saridakis and S. Chakraborty, Observational constraints on one-parameter dynamical dark-energy parametrizations and the $H_{0}$ tension, Phys. Rev. D 99, no.4, 043543 (2019) [arXiv: 1810.05141$].$

[55] F. K. Anagnostopoulos, S. Basilakos and E. N. Saridakis, Bayesian analysis of $f(T)$ gravity using $f \sigma_{8}$ data, Phys. Rev. D 100, no.8, 083517 (2019) [arXiv: 1907. 07533].

[56] S. Pan, W. Yang, E. Di Valentino, E. N. Saridakis and S. Chakraborty, Interacting scenarios with dynamical dark energy: Observational constraints and alleviation of the $H_{0}$ tension, Phys. Rev. D 100, no.10, 103520 (2019) [arXiv: 1907.07540].

[57] F. K. Anagnostopoulos, S. Basilakos and E. N. Saridakis, Observational constraints on Barrow holographic dark energy, [arXiv:2005.10302].

[58] J. Alfaro, M. San Martín and C. Rubio, Observational constraints in Delta Gravity: CMB and supernovas, [arXiv:2009.13305].

[59] F. Felegary, I. A. Akhlaghi and H. Haghi, Evolution of matter perturbations and observational constraints on tachyon scalar field model, Phys. Dark Univ. 30, 100739 (2020). 
[60] E. Di Valentino, $A$ (brave) combined analysis of the $H_{0}$ late time direct measurements and the impact on the Dark Energy sector, [arXiv:2011.00246].

[61] A. Paliathanasis, S. Basilakos, E. N. Saridakis, S. Capozziello, K. Atazadeh, F. Darabi and M. Tsamparlis, New Schwarzschild-like solutions in $f(T)$ gravity through Noether symmetries, Phys. Rev. D 89, 104042 (2014) [arXiv: 1402 . 5935].

[62] A. Paliathanasis, $f(R)$-gravity from Killing Tensors, Class. Quant. Grav. 33, no. 7, 075012 (2016) [arXiv:1512.03239].

[63] N. Dimakis, A. Karagiorgos, A. Zampeli, A. Paliathanasis, T. Christodoulakis and P. A. Terzis, General Analytic Solutions of Scalar Field Cosmology with Arbitrary Potential, Phys. Rev. D 93, no. 12, 123518 (2016) [arXiv: 1604 .05168].

[64] H. Yu, B. Ratra and F. Y. Wang, Hubble Parameter and Baryon Acoustic Oscillation Measurement Constraints on the Hubble Constant, the Deviation from the Spatially Flat $\Lambda C D M$ Model, the Deceleration/Acceleration Transition Redshift, and Spatial Curvature, Astrophys. J. 856, no. 1, 3 (2018) [arXiv:1711.03437].

[65] R. Kessler and D. Scolnic, in Photometrically Identified Samples," Astrophys. J. 836, no.1, 56 (2017) doi:10.3847/1538-4357/836/1/56 [arXiv:1610.04677 [astro-ph.CO]].

[66] M. Moresco, R. Jimenez, L. Verde, L. Pozzetti, A. Cimatti and A. Citro, Setting the Stage for Cosmic Chronometers. I. Assessing the Impact of Young Stellar Populations on Hubble Parameter Measurements, Astrophys. J. 868, no. 2, 84 (2018) [arXiv:1804.05864].

[67] D. M. Scolnic, D. O. Jones, A. Rest, Y. C. Pan, R. Chornock, R. J. Foley, M. E. Huber, R. Kessler, G. Narayan and A. G. Riess, et al. The Complete Light-curve Sample of Spectroscopically Confirmed SNe Ia from Pan-STARRSI and Cosmological Constraints from the Combined Pantheon Sample, Astrophys. J. 859, no.2, 101 (2018) [arXiv:1710.00845].

[68] D. J. Eisenstein and W. Hu, Baryonic features in the matter transfer function, Astrophys. J. 496 (1998), 605 [arXiv:astro-ph/9709112].

[69] S. Alam et al. [eBOSS], The Completed SDSS-IV extended Baryon Oscillation Spectroscopic Survey: Cosmological Implications from two Decades of Spectroscopic Surveys at the Apache Point observatory, [arXiv:2007.08991].

[70] J. Ryan, Y. Chen and B. Ratra, Baryon acoustic oscillation, Hubble parameter, and angular size measurement constraints on the Hubble constant, dark energy dynamics, and spatial curvature, Mon. Not. Roy. Astron. Soc. 488, no.3, 3844-3856 (2019) [arXiv: 1902 . 03196].

[71] D. F. Torres, H. Vucetich and A. Plastino, Early universe test of nonextensive statistics, Phys. Rev. Lett. 79, 1588-1590 (1997) [erratum: Phys. Rev. Lett. 80, 3889 (1998)] [arXiv:astro-ph/9705068].

[72] G. Lambiase, Lorentz invariance breakdown and constraints from big-bang nucleosynthesis, Phys. Rev. D 72, 087702 (2005) [arXiv: astro-ph/0510386].

[73] J. D. Barrow, S. Basilakos and E. N. Saridakis, Big Bang Nucleosynthesis constraints on Barrow entropy, [arXiv:2010.00986].

[74] N. Aghanim et al. [Planck], Planck 2018 results. VI. Cosmological parameters, Astron. Astrophys. 641 (2020), A6 [arXiv:1807.06209].

[75] J. Goodman and J. Weare, Ensemble samplers with affine invariance, Comm. App. Math. and Comp. Sci. 5, 65 (2010). 
[76] D. Foreman-Mackey, D. W. Hogg, D. Lang and J. Goodman, emcee: The MCMC Hammer, [arXiv:1202.3665].

[77] H. Akaike, A new look at the statistical model identification, IEEE Transactions on Automatic Control, 19, 716, (1974).

[78] G. Schwarz, Estimating the Dimension of a Model Ann. Statist., 6, 2, 461 (1978).

[79] D. J. Spiegelhalter, N. G. Best, B. P. Carlin, A. Van Der Linde, Bayesian measures of model complexity and fit Jour. of the R. Stat. Soc., 64 4, 583 (2002).

[80] R. Andrae, T. Schulze-Hartung and P. Melchior, Dos and don'ts of reduced chi-squared, [arXiv:1012.3754].

[81] K. Anderson, Model selection and multimodel inference: a practical information-theoretic approach, 2nd edn. Springer, New York (2002).

[82] K. P. Burnham, D. R. Anderson Multimodel Inference: Understanding AIC and BIC in Model Selection Sociological Methods and Research 33, 261 (2004).

[83] A. R. Liddle, Information criteria for astrophysical model selection, Mon. Not. Roy. Astron. Soc. 377, (2007) L74, [arXiv: astro-ph/0701113].

[84] R. E. Kass and A. E. Raftery, Bayes Factors, J. Am. Statist. Assoc. 90, no. 430, 773 (1995).

[85] S. Cao, J. Ryan and B. Ratra, Using Pantheon and DES supernova, baryon acoustic oscillation, and Hubble parameter data to constrain the Hubble constant, dark energy dynamics, and spatial curvature, [arXiv:2101.08817].

[86] A. G. Riess et al., A 2.4\% Determination of the Local Value of the Hubble Constant, Astrophys. J. 826, no. 1, 56 (2016) [arXiv: 1604.01424 ].

[87] S. Birrer, A. J. Shajib, A. Galan, M. Millon, T. Treu, A. Agnello, M. Auger, G. C. F. Chen, L. Christensen and T. Collett, et al. TDCOSMO - IV. Hierarchical time-delay cosmography - joint inference of the Hubble constant and galaxy density profiles, Astron. Astrophys. 643, A165 (2020) [arXiv:2007.02941].

[88] W. L. Freedman, B. F. Madore, T. Hoyt, I. S. Jang, R. Beaton, M. G. Lee, A. Monson, J. Neeley and J. Rich, Calibration of the Tip of the Red Giant Branch (TRGB), [arXiv:2002.01550].

[89] G. Chen and B. Ratra, Median statistics and the Hubble constant, Publ. Astron. Soc. Pac. 123, 1127-1132 (2011) [arXiv:1105.5206].

[90] M. Rigault, G. Aldering, M. Kowalski, Y. Copin, P. Antilogus, C. Aragon, S. Bailey, C. Baltay, D. Baugh and S. Bongard, et al. Confirmation of a Star Formation Bias in Type Ia Supernova Distances and its Effect on Measurement of the Hubble Constant, Astrophys. J. 802, no.1, 20 (2015) [arXiv:1412.6501].

[91] Y. Chen, S. Kumar and B. Ratra, Determining the Hubble constant from Hubble parameter measurements, Astrophys. J. 835, no.1, 86 (2017) [arXiv: 1606 . 07316].

[92] B. R. Zhang, M. J. Childress, T. M. Davis, N. V. Karpenka, C. Lidman, B. P. Schmidt and M. Smith, A blinded determination of $H_{0}$ from low-redshift Type Ia supernovae, calibrated by Cepheid variables, Mon. Not. Roy. Astron. Soc. 471, no.2, 2254-2285 (2017) [arXiv:1706. 07573].

[93] S. Dhawan, S. W. Jha and B. Leibundgut, Measuring the Hubble constant with Type Ia supernovae as near-infrared standard candles, Astron. Astrophys. 609, A72 (2018) [arXiv: 1707.00715 ].

[94] D. Fernández Arenas, E. Terlevich, R. Terlevich, J. Melnick, R. Chávez, F. Bresolin, E. Telles, 
M. Plionis and S. Basilakos, An independent determination of the local Hubble constant, Mon. Not. Roy. Astron. Soc. 474, no.1, 1250-1276 (2018) [arXiv: 1710 . 05951].

[95] K. Blum, E. Castorina and M. Simonović, Could Quasar Lensing Time Delays Hint to a Core Component in Halos, Instead of $H_{0}$ Tension?, Astrophys. J. Lett. 892, no.2, L27 (2020) [arXiv:2001.07182].

[96] O. Farooq, F. R. Madiyar, S. Crandall and B. Ratra, Hubble Parameter Measurement Constraints on the Redshift of the Deceleration-acceleration Transition, Dynamical Dark Energy, and Space Curvature, Astrophys. J. 835, no.1, 26 (2017) [arXiv: 1607 . 03537].

[97] M. Rezaei, S. Pour-Ojaghi and M. Malekjani, A Cosmography Approach to Dark Energy Cosmologies: New Constraints Using the Hubble Diagrams of Supernovae, Quasars, and Gamma-Ray Bursts, Astrophys. J. 900, no.1, 70 (2020) [arXiv: 2008 . 03092].

[98] B. S. Haridasu, V. V. Luković, M. Moresco and N. Vittorio, An improved model-independent assessment of the late-time cosmic expansion, JCAP 10 (2018), 015 [arXiv: 1805.03595 ]. 